\title{
Coincident Visualization of Uncertainty and Value for Point Symbols
}

\author{
Petr Kubíček ${ }^{\mathrm{a}}$ *, Milan Konečný ${ }^{\mathrm{a}}$, Jie Shen ${ }^{\mathrm{b}}$, Zdeněk Stachoň ${ }^{\mathrm{a}}$, Radim Štampach ${ }^{\mathrm{a}}$, Xinqian \\ $\mathrm{Wu}^{\mathrm{b}}$, Lukáš Herman ${ }^{\mathrm{a}}$, Karel Staněk ${ }^{\mathrm{a}}$, Tomáš Řezník ${ }^{\mathrm{a}}$ \\ ${ }^{a}$ Department of Geography, Faculty of Science, Masaryk University, Kotlářská 2, 61137 Brno, Czech Republic, \\ kubicek@geogr.muni.cz,konecny@geogr.muni.cz,zstachon@geogr.muni.cz,r.stampach@centrum.cz,herman.lu@mail.muni.cz, \\ karst@geogr.muni.cz,tom@mail.muni.cz. \\ ${ }^{b}$ School of Geographic Science, Nanjing Normal University, Nanjing, Jiangsu 210023, China, shenjie@njnu.edu.cn, \\ 446196400@qq.com \\ * Corresponding author
}

Keywords: Uncertainty, Bivariate visualization, Intercultural comparison

Abstract:

The issue of uncertainty as a generic phenomenon in the natural world has been at the centre of both the cartographic and GI communities since the beginning of geographic data quality research. In accordance with the development of theoretical aspects of cartographic visualization and methods of uncertainty propagation in models, the generally accepted opinion is that uncertainty has to be presented to users in an unambiguous and understandable way. Despite reasonable amounts of work done in the field of uncertainty visualization methods (MacEachren1992, Leitner and Buttenfield 2000) and the testing of impact of visualization on decision making (Senaratne et al. 2012; Kinkeldy et al. 2015), there is still a wide gap between the uncertainty visualization theory and widely accepted use of uncertainty representation within decision making process. MacEachren et al. (2012), Fabrikant et al. (2010) initiated the discussion towards optimization of uncertainty visualization regarding visual semiotics and use of specific representations of uncertainty within complex mapping compositions and application context. However, their studies left also some open questions to be solved regarding the international audience of users.

The presented study focused on two unresolved topics, namely how would users perceive the uncertainty point map signs within a complex map field and what would be the appropriate visualization in case if there is a need to combine value and uncertainty together. Moreover, we performed the testing in two different cultural environments in Brno (Czech Republic, Europe) and Nanjing (China).

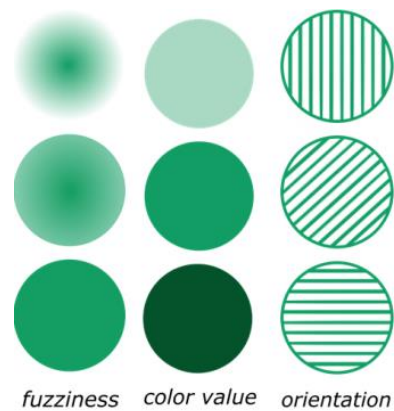

a

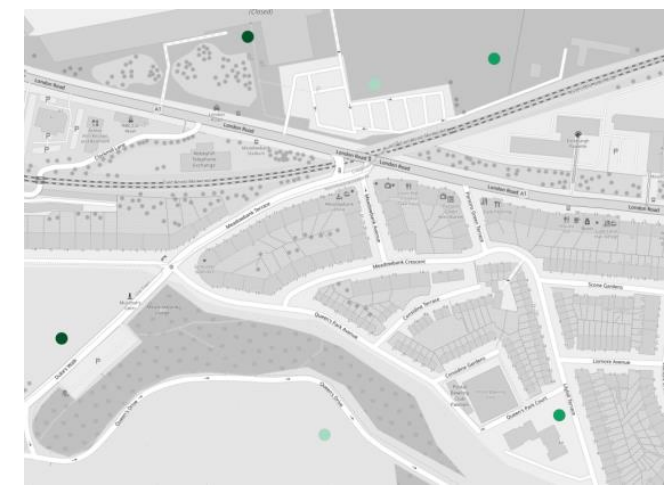

b

Figure 1. Visual variables from MacEachren et al. (2012) used for the intuitiveness testing - stand-alone (a) and used in a map display (b).

At the beginning, we replicated the empirical study performed by MacEachren et al (2012) and asked participants to rate on a scale of 1-7 (Likert) the intuitiveness of a sign set to represent a category of attribute uncertainty (fig. 1a). Based on the results of MacEachren et al. (2012) we used two visual variables with high intuitiveness (fuzziness and colour value) and one control variable scoring below average (orientation). In the following six tasks we used the point signs in the map field (fig. 1b) asked users to estimate the certainty/uncertainty of the selected sign.

Both data value and its attribute uncertainty are often visualized together in a form of bivariate visualization. In the second part of the test visual (bivariate) signs represented environmental sensors measurements and their uncertainty (fig. 3). We used the combination of size and value for one set of signs and size and fuzziness for the other. 


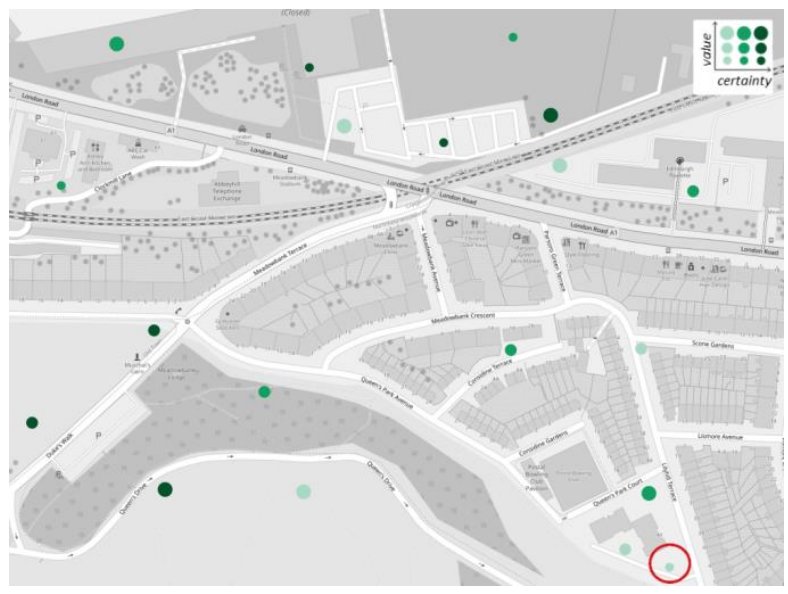

Figure 3. Bivariate visualization (value $\mathrm{x}$ size) used for the depiction of value and uncertainty in a single map sign.

Users were asked to identify a single value or uncertainty level of the selected sign (six tasks) and then a combination of both variables (value and uncertainty together, six tasks). The final section was focused on the ability of users to identify not only a single sign but also a simple pattern. We marked four areas on the map and asked the users to choose the area with a specific spatial pattern (least certain with high values, fig. 4).

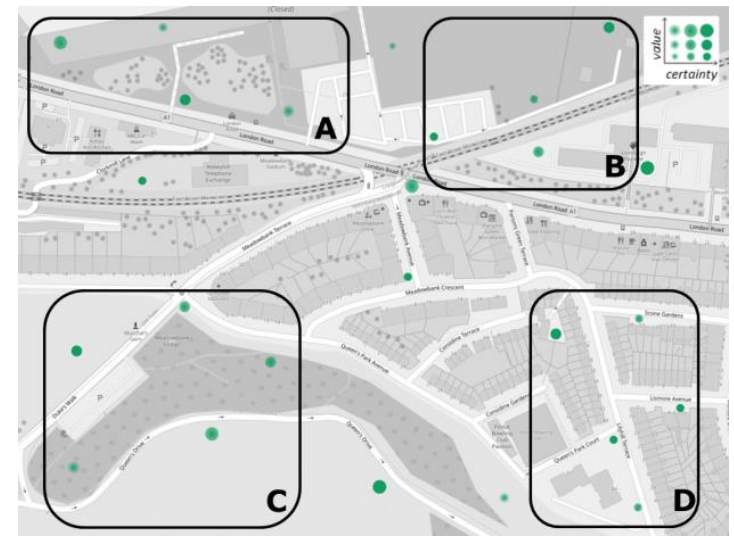

Figure 4. Identification of a specific spatial pattern of value and uncertainty.

The Czech part of the testing is still going on, however, the preliminary results (70 participants) from China confirm the high intuitiveness of both value and fuzziness and low performance of orientation. The results for a map composition and bivariate visualization are pending and will be presented at the conference. 